\title{
Invisible Higgs in weak bosons associative production with heavy quarks at LHC
}

\author{
S. V. Demidov* \\ Institute for Nuclear Research of the Russian Academy of Sciences, 60th October Anniversary \\ prospect 7a, Moscow 117312, Russia \\ E-mail: demidovems2.inr.ac.ru

\section{E. E. Boos} \\ Skobeltsyn Institute of Nuclear Physics, Moscow State University, Vorobiovy gory, Moscow \\ 119991, Russia \\ E-mail: boos@theory.sinp.msu.ru \\ D. S. Gorbunov \\ Institute for Nuclear Research of the Russian Academy of Sciences, 60th October Anniversary \\ prospect 7a, Moscow 117312, Russia \\ E-mail: gorby@ms2.inr.ac.ru
}

\begin{abstract}
We consider class of models where the dominating Higgs boson decay modes are invisible. A common way to search for the Higgs boson in these models is to look for missing $p_{T}$ signature. However, this leaves the width of the Higgs boson unobservable. We propose a new strategy to search for invisible Higgs which can be used for extracting both the Higgs boson mass and width.
\end{abstract}

The XIXth International Workshop on High Energy Physics and Quantum Field Theory, QFTHEP2010 September 08-15, 2010

Golitsyno, Moscow, Russia

\footnotetext{
* Speaker.
} 


\section{Introduction}

The Standard Model describes accurately almost all phenomena in particle physics and the only particle which has not been observed so far within the SM is the Higgs boson. However, it has become clear that the SM itself can not be complete. There are neutrino oscillations, strong CPproblem, dark matter, baryon asymmetry of the Universe which lack for explanation within the SM (see, e.g. review section of Ref. [1]). It can happen that the physics responsible for electroweak symmetry breaking can also be related to these phenomena. Among such extensions of the SM there are classes of models in which the properties of the Higgs boson (in particular, its decay pattern) get modified (see, e.g. [2]-[阿). Here we consider the possibility that a new invisible Higgs boson decay mode dominates.

The strategy for hunting the invisible Higgs boson at LHC is to search for missing $P_{T}$ events in various channels such as Vector Boson Fusion $q q \rightarrow q q H$ [10], the associated production processes, $g g \rightarrow t \bar{t} H$ [11] and $q q \rightarrow Z H$ or $q q \rightarrow W^{ \pm} H$ [12, 13]. A tricky question here is how to make sure that the observed signal is really due to production of the Higgs boson, not some other particle. Another disadvantage of the missing $P_{T}$ signature is that only the Higgs boson mass can be estimated from the data analysis: the Higgs boson width remains unobservable.

We propose to use the channels $p p \rightarrow Z Z \bar{t} t, p p \rightarrow W W \bar{t} t$ and $p p \rightarrow Z Z \bar{b} b, p p \rightarrow W W \bar{b} b$ to extract the properties of the invisible Higgs boson in the mass interval $130-180 \mathrm{GeV}$. The reason is that the exchange of the Higgs boson in subdiagramms of these processes corresponding to heavy fermion-antifermion scattering into massive vector bosons should be considerable because it restores the unitarity [14, 16, 15]. In case of the Higgs boson mass above the weak boson pair thresholds these processes have been thoroughly studied [17]. The virtual Higgs boson contributions to $W^{+} W^{-}$and $Z Z$ production via weak boson fusion and gluon fusion have been also considered in literature, see, e.g., [18]. The main observation of our work [22] is that the measurements of total cross section and invariant mass distribution of the weak boson pair generally allow to estimate both the Higgs boson mass and width.

\section{Invisible Higgs in $p p \rightarrow t \bar{t} Z Z$ and $p p \rightarrow b \bar{b} Z Z$ at LHC}

We would like to consider virtual contribution of the Higgs boson to the process $p p \rightarrow t \bar{t} Z Z$, since it remains almost the same as in the SM (we consider the case where all couplings of the Higgs boson to the SM fields are intact). We use CompHEP [19-21] to calculate the tree level partonic cross sections of this process (details of calculations can be found in Ref. [22]). Note, that we take into account both diagramms with and without the Higgs boson.

The results for total cross sections and invariant mass $Z$-boson pair distributions are presented in Figures 1-3. In Figure 1 1 we plot the dependence of the total cross section of $p p \rightarrow t \bar{t} Z Z$ channel at $\sqrt{s}=14 \mathrm{TeV}$ and $\sqrt{s}=10 \mathrm{TeV}$ on the Higgs boson mass $m_{H}$ for a set of values of the Higgs boson width $\Gamma_{H}$. As one expects, at large values of the Higgs boson width the virtual Higgs boson contribution to the amplitude of this process decreases and hence the total cross section also decreases. Figure 2 shows the corresponding invariant mass $m_{Z Z}$ distribution for different values of Higgs boson mass in the cases of the SM Higgs boson width (upper panel) and eight times larger width (lower panel). We see that both shape and position of maximum of $m_{Z Z}$ distribution 

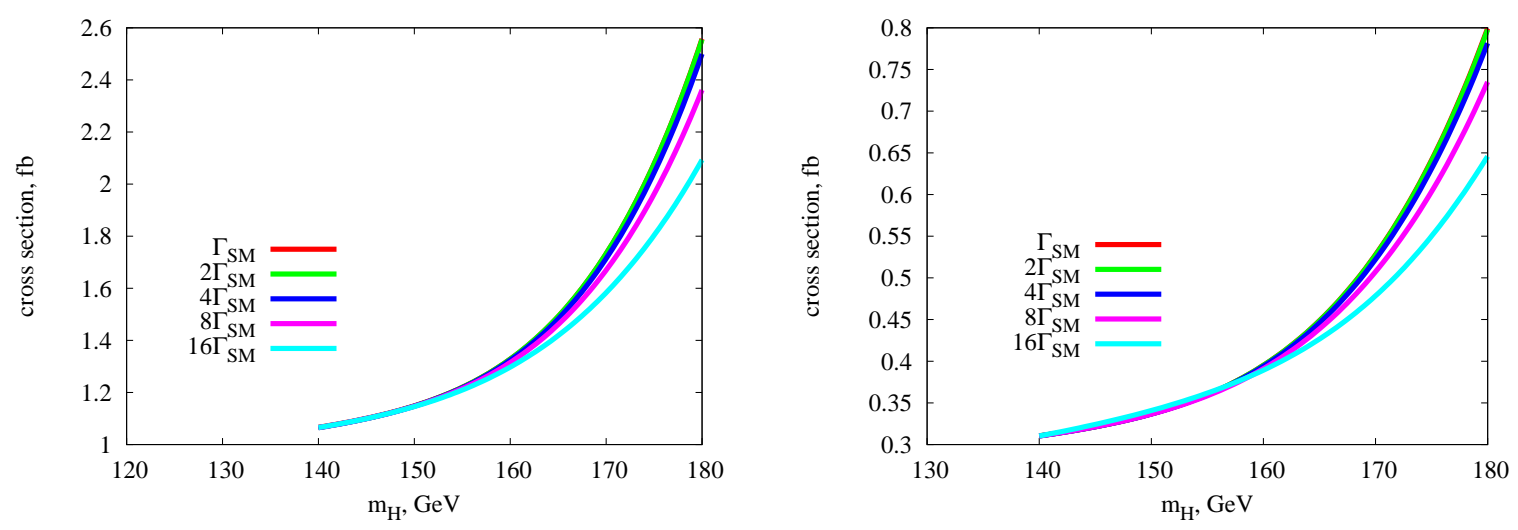

Figure 1: The dependence of the total cross section $p p \rightarrow t \bar{t} Z Z$ at $\sqrt{s}=14 \mathrm{TeV}$ (left panel) and $\sqrt{s}=10 \mathrm{TeV}$ (right panel) on the mass of the Higgs boson in for a set of values of the Higgs boson width. Here $\Gamma_{S M}$ is the width of the Standard Model Higgs boson.
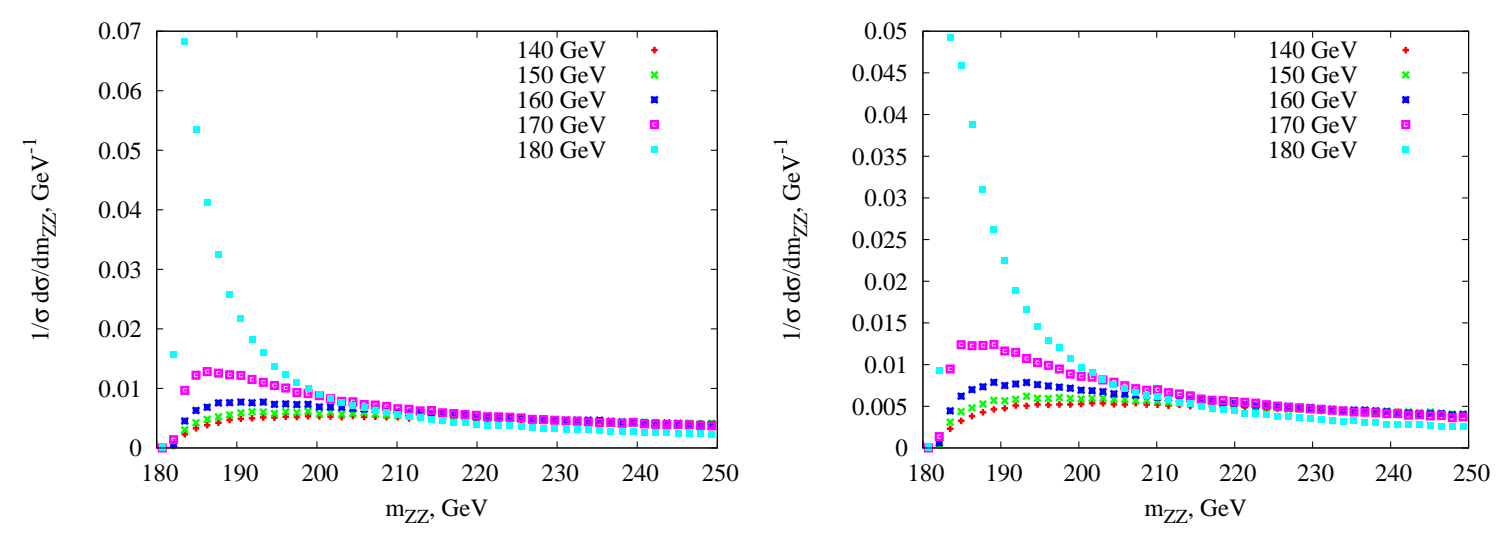

Figure 2: The invariant mass $m_{Z Z}$ distribution for the $p p \rightarrow t \overline{t Z Z}$ at $\sqrt{s}=14 \mathrm{TeV}$ process for several values of the Higgs boson mass for Standard Model Higgs width (upper panel) and for the width which is 8 times larger (lower panel).

strongly depend on mass $m_{H}$, which can be used to pin down the Higgs boson mass. Moreover, from Figure 3 one concludes that this $m_{Z Z}$ distribution does not depend on the width of the Higgs boson, except for the case of near threshold values of its mass (see lower panel). Even in the latter case, for $m_{H}$ near $180 \mathrm{GeV}$, we observe that the position of maximum in $m_{Z Z}$ distribution varies quite moderately with reasonable increase of the Higgs boson width. Parameters of the Higgs boson - mass and width - can be obtained, as usual, from the combined two-parametric fit to the observables of this channels.

It is worth noting that the total cross sections of the processes with $t$-quarks considered in the previous section are of order of a few $\mathrm{fb}$, which requires high luminosity running of LHC to be of practical interest. The same is true for similar channels $p p \rightarrow b \bar{b} Z Z$ and $p p \rightarrow b \bar{b} W^{+} W^{-}$ within the SM because the Yukawa coupling of $b$-quarks to Higgs boson is quite small. However, in many promising extensions of the SM this Yukawa coupling increases. For illustrative purposes we take the Yukawa coupling of $b$-quarks increased by a factor $A=50$ with respect to the SM case. 

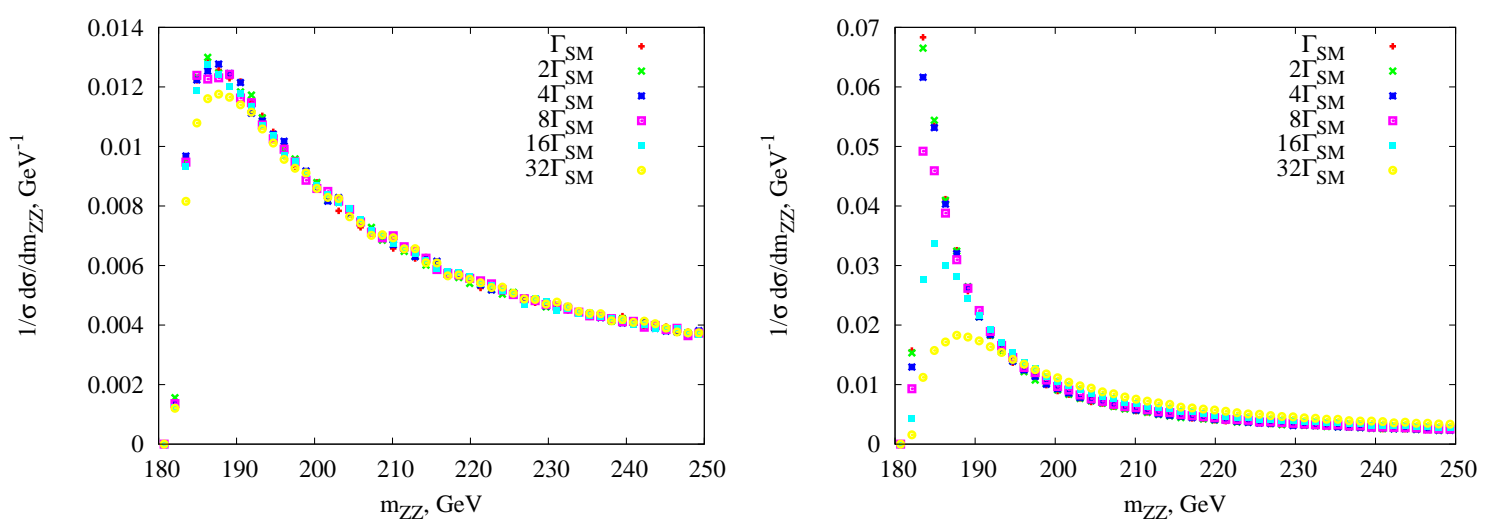

Figure 3: The dependence of the $m_{Z Z}$ invariant mass distribution in $p p \rightarrow t \bar{t} Z Z$ on the width of the Higgs boson for the following values of masses $m_{H}: 170 \mathrm{GeV}$ (top panel), $180 \mathrm{GeV}$ (bottom panel), at $\sqrt{s}=14 \mathrm{TeV}$.

Note, that the change of the $b$-quark Yukawa coupling also yields a change of the Higgs boson total width which we take into account accordingly. In these modifications of the SM with large value of $A$ the Higgs boson width $\Gamma_{m S M}$ is saturated by its decay into $b$-quarks. For the processes with $b$-quarks we exclude from considerations the following regions of the phase space of the final state: $159.3 \mathrm{GeV}<m_{b W^{-}}<189.3 \mathrm{GeV}$ and $159.3 \mathrm{GeV}<m_{\bar{b} W^{+}}<189.3 \mathrm{GeV}$ because in these regions the cross section is saturated by top-quark production and the interesting effects get obscured. The corresponding total cross sections are given in Figure $\bigoplus$ for $b \bar{b} Z Z$ final state.
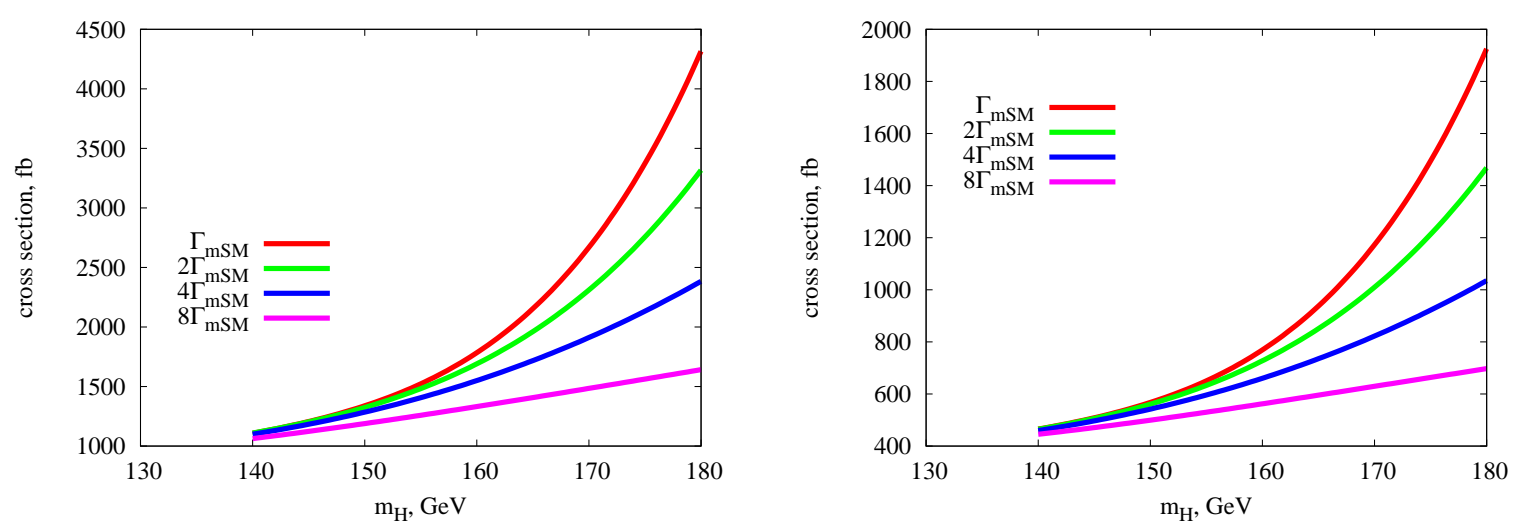

Figure 4: The dependence of the total cross section of $p p \rightarrow b \bar{b} Z Z$ at $\sqrt{s}=14 \mathrm{TeV}$ (left) and $\sqrt{s}=10 \mathrm{TeV}$ (right) on the mass of the Higgs boson in the modified Standard Model with b-Higgs coupling enhanced by factor $A=50$.

For the processes with $b$-quarks we observe qualitatively similar dependence of the $m_{Z Z}$ distribution on the Higgs boson mass; remarkably, this distribution depends also on the Higgs boson width, see Figures 5 and 6 . However, both the shape of $m_{Z Z}$ distribution and the position of its maximum are much more sensitive to $m_{H}$ and $\Gamma_{H}$ as compared to the case of $t$-quarks. So, to obtain the Higgs boson width and mass one should make two-parametric analysis of $m_{Z Z}$ distribution and total cross section. At the same time, with the same collected statistics one can expect to achieve 
higher accuracy in measurements of the Higgs boson mass and width, than in the channels with $t$-quarks. Of course this is true only for large enough values for the constant A.
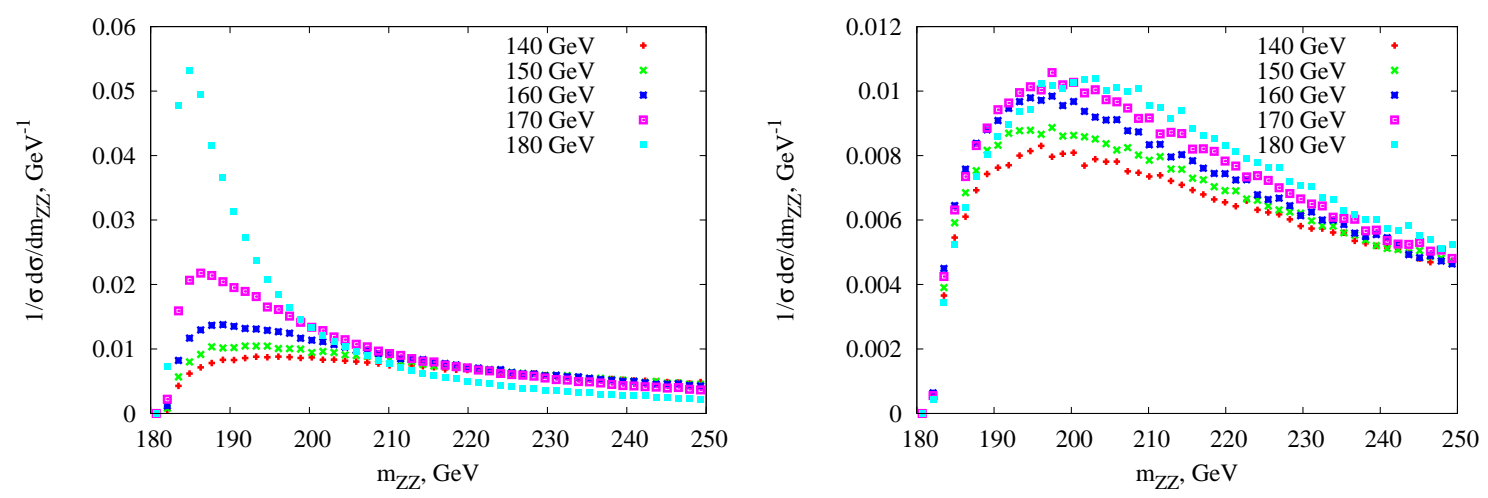

Figure 5: The invariant mass $m_{Z Z}$ distribution in the $p p \rightarrow b \bar{b} Z Z$ process for several values of the Higgs boson mass for modified Standard Model Higgs boson width $\Gamma_{m S M}$ (upper panel) and for the width 8 times larger due to invisible decay mode (lower panel).
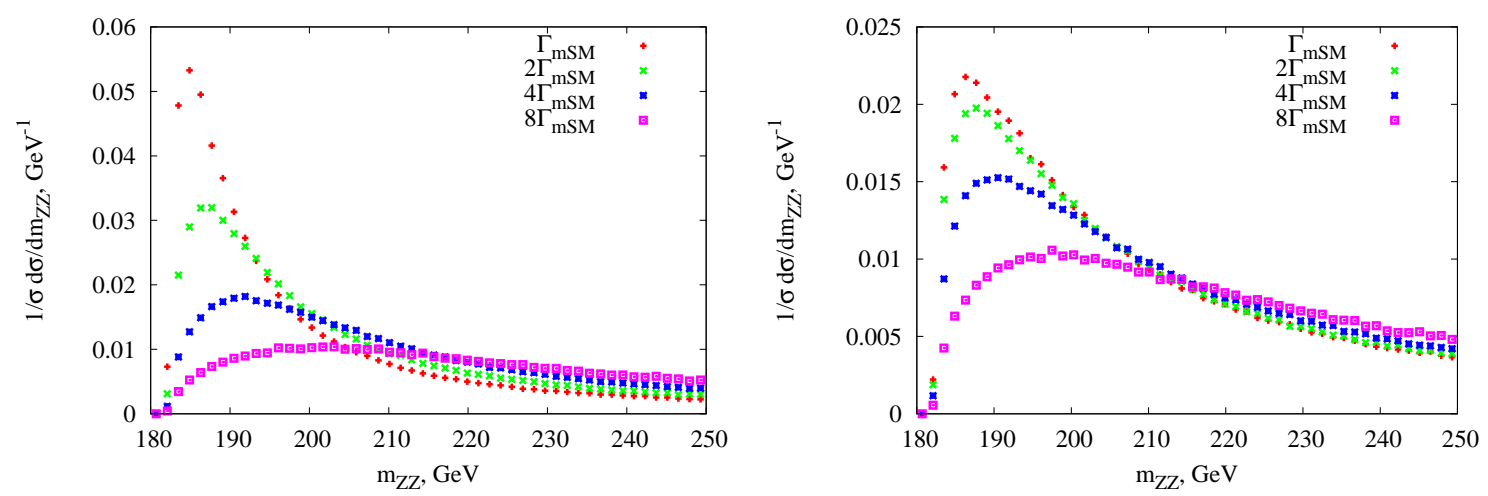

Figure 6: The dependence of the total cross section of $p p \rightarrow b \bar{b} Z Z$ on the width of the Higgs boson for $m_{H}=180 \mathrm{GeV}, \Gamma_{m S M}=9.04 \mathrm{GeV}$ (upper panel) and $m_{H}=170 \mathrm{GeV}, \Gamma_{m S M}=8.41 \mathrm{GeV}$ (lower panel).

Note, that all our calculations have been done at the leading order in perturbative QCD. We have performed a simple estimate of next QCD corrections by introducing changes in renormalization scale. Our results show that in accordance with known NLO computations for the Higgs production in association with heavy quarks the cross sections and distributions get corrections, however, the observed dependence on the Higgs mass and width is not practically affected [22].

One can also investigate another similar channels, $p p \rightarrow t \bar{t} W^{+} W^{-}$and $p p \rightarrow b \bar{b} W^{+} W^{-}$. The behaviour of the total cross sections and the invariant mass distribution for massive vector bosons are similar to that of for processes with top-quarks [22].

Acknowledgments. The work was supported by Russian Ministry of Education and Science under state contract 02.740 .11 .0244 . The work of E.B. was also supported by the grant of Russian Ministry of Education and Science NS-4142.2010.2, RFBR grants 08-02-91002-CERN_a and 0802-92499-CNRSL_a. D.G. thanks the organizers of the long-term workshop in Yukawa Institute 
YITP-T-10-01 for hospitality. The work of D.G. and S.D. was supported in part by the Russian Foundation for Basic Research (grants 08-02-00473a), by the grants of the President of the Russian Federation NS-5525.2010.2 and by FAE program (government contract П520). The work of S.D. was also supported by the grants of the President of the Russian Federation MK-4317.2009.2, by FAE program (government contract 12598 ). Numerical part of the work was performed on the Computational cluster of the Theoretical Division of INR RAS.

\section{References}

[1] C. Amsler et al. [Particle Data Group], Phys. Lett. B 667, 1 (2008).

[2] B. Patt and F. Wilczek, "Higgs-field portal into hidden sectors," arXiv:hep-ph/0605188.

[3] R. E. Shrock and M. Suzuki, Phys. Lett. B 110 (1982) 250.

[4] N. Arkani-Hamed, S. Dimopoulos and G. R. Dvali, Phys. Lett. B 429, 263 (1998) [arXiv:hep-ph/9803315].

[5] L. Randall and R. Sundrum, Phys. Rev. Lett. 83 (1999) 3370 [arXiv:hep-ph/9905221].

[6] K. Belotsky, D. Fargion, M. Khlopov, R. Konoplich and K. Shibaev, Phys. Rev. D 68 (2003) 054027 [arXiv:hep-ph/0210153].

[7] N. V. Krasnikov, Mod. Phys. Lett. A 13 (1998) 893 [arXiv:hep-ph/9709467].

[8] Y. Hosotani, K. Oda, T. Ohnuma and Y. Sakamura, Phys. Rev. D 78 (2008) 096002 [Erratum-ibid. D 79 (2009) 079902] [arXiv:0806.0480 [hep-ph]].

[9] L. Fromme, S. J. Huber and M. Seniuch, JHEP 0611, 038 (2006) [arXiv:hep-ph/0605242].

[10] O. J. P. Eboli and D. Zeppenfeld, Phys. Lett. B 495 (2000) 147 [arXiv:hep-ph/0009158].

[11] J. F. Gunion, Phys. Rev. Lett. 72 (1994) 199 [arXiv:hep-ph/9309216].

[12] D. Choudhury and D. P. Roy, Phys. Lett. B 322 (1994) 368 [arXiv:hep-ph/9312347].

[13] R. M. Godbole, M. Guchait, K. Mazumdar, S. Moretti and D. P. Roy, Phys. Lett. B 571 (2003) 184 [arXiv:hep-ph/0304137].

[14] B. W. Lee, C. Quigg and H. B. Thacker, Phys. Rev. D 16, 1519 (1977).

[15] T. Appelquist and M. S. Chanowitz, Phys. Rev. Lett. 59, 2405 (1987) [Erratum-ibid. 60, 1589 (1988)].

[16] M. S. Chanowitz, M. A. Furman and I. Hinchliffe, Nucl. Phys. B 153, 402 (1979).

[17] J. Bagger et al., Phys. Rev. D 52, 3878 (1995) [arXiv:hep-ph/9504426].

[18] H. Davoudiasl, T. Han and H. E. Logan, Phys. Rev. D 71, 115007 (2005) [arXiv:hep-ph/0412269].

[19] A. Pukhov et al., "CompHEP: A package for evaluation of Feynman diagrams and integration over multi-particle phase space. User's manual for version 33," arXiv:hep-ph/9908288.

[20] E. Boos et al. [CompHEP Collaboration], Nucl. Instrum. Meth. A 534 (2004) 250 [arXiv:hep-ph/0403113].

[21] http://comphep.sinp.msu.ru/

[22] E. E. Boos, S. V. Demidov and D. S. Gorbunov, arXiv:1010.5373 [hep-ph]. 